\title{
Curcumin Effect on the Prevention of Gingival Overgrowth Following Phenytoin Consumption in Rats: A Clinicohistological and Immunohistochemical Study
}

\author{
Shahriar Eftekharian ${ }^{1}$, Safoura Seifi ${ }^{2}$, Farhad D Satari ${ }^{3}$, Ali Akbar Moghaddamnia ${ }^{4}$, Farideh Feizi ${ }^{5}$, Sohrab Kazemi ${ }^{6}$,
} Hemmat Gholinia ${ }^{7}$

\begin{abstract}
Aim: At the moment there is no clear evidence with clinico-histological and immunohistochemical studies in animals to show the curcumin effect on the gingival overgrowth following phenytoin consumption. The purpose of the present study was to identify this subject.

Materials and methods: In this experimental study, 50 adult male Wistar rats were divided into three groups. The rats in groups I and II received $100 \mathrm{mg} / \mathrm{kg}$ of phenytoin per day. Group II also received $20 \mathrm{mg} / \mathrm{kg}$ intraperitoneal curcumin per day. The control group received the curcumin vehicle only. Gingival clinical dimensions were measured at the beginning and end of the study. The rats were then sacrificed, biopsy of gingiva was prepared, and the samples were stained with hematoxylin-eosin. Morphometry was performed to evaluate the degree of inflammation, epithelial thickness, number, and cross-sectional area of the blood vessels. Immunohistochemical staining was performed using Ki67 and a-SMA. Results: Compared to the control group, Phenytoin in group I increased gingival volume. There was significance difference in group II with group I and control after intervention in the clinical view $(p=0.002)$. The difference in the number of blood vessels between groups I and II was statistically significant $(p=0.001)$. Significant differences were observed in blood vessel cross-sectional area $(p=0.001)$, epithelial thickness $(p=0.002)$, Ki67, and a-SMA expression between groups I and II $(p=0.001)$.

Conclusion: In rats, curcumin seems to exerts its effects in preventing an increase in gingival volume caused by Phenytoin through decreasing the inflammatory infiltration, decreasing the number of blood vessels and increasing their cross-sectional area, decreasing the thickness of the epithelium, and decreasing the expression of Ki67 and a-SMA.

Clinical significance: It is suggested that curcumin may be effective in treatment of gingival enlargement following Phenytoin consumption in future. Larger sample size and clinical trials study are recommended.

Keywords: a-SMA, Curcumin, Gingival overgrowth, Immunohistochemistry, Ki67, Morphometry, Phenytoin.

The Journal of Contemporary Dental Practice (2019): 10.5005/jp-journals-10024-2653
\end{abstract}

\section{INTRODUCTION}

Gingival enlargement following medication use can lead to difficulties in speaking, chewing, face esthetic, and teeth eruption. Clinical and microscopic features of this type of increase in volume caused by different medications are similar. Phenytoin (dilantin) is an anti-seizure medication, which belongs to the Hydantoin family and results in gingival enlargement in about $50 \%$ of the patients. ${ }^{1}$ The cellular and molecular mechanisms of the Phenytoin effect have not been precisely known.

Phenytoin has a similar, but stronger, increasing effect on PGE2 levels like proinflammatory cytokines and, as a result, increases gingival volume, in which PGE2 increases the expression of the connective tissue growth factor via EP3 receptors (prostaglandin E receptor 3 ) and the JNK (c-jun NH2-terminal kinase) enzyme. ${ }^{2}$

Curcumin is an active ingredient derived from turmeric, with various biological activities such as antitumor and antiinflammatory properties. Studies on rats have shown that curcumin significantly reduces inflammatory infiltration and increases the collagen content and the number of fibroblasts. Curcumin prevents the expression of cytokines such as IL- 6 and TNF- $a$ at the protein and mRNA level and decreases the expression of the connective tissue growth factor (CCN2) derived from thrombin by inhibiting the JNK receptor in human gingival fibroblasts. ${ }^{3}$

Yang et al. evaluated the effect of curcumin on cultured human fibroblasts and reported that curcumin reduces TGF- $\beta$-induced
${ }^{1}$ Babol University of Medical Sciences, Babol, Iran

${ }^{2}$ Department of Oral and Maxillofacial Pathology, Babol University of Medical Sciences, Babol, Iran

${ }^{3}$ Department of Periodontology, Babol University of Medical Sciences, Babol, Iran

${ }^{4,6}$ Department of Pharmacology, Babol University of Medical Sciences, Babol, Iran

${ }^{5}$ Department of Oral Histology, Babol University of Medical Sciences, Babol, Iran

${ }^{7}$ Department of Epidemiology and Statics, Babol University of Medical Sciences, Babol, Iran

Corresponding Author: Safoura Seifi, Department of Oral and Maxillofacial Pathology, Babol University of Medical Sciences, Babol, Iran, Phone: +98 11-32291408-9, e-mail: sf_seify@yahoo.com

How to cite this article: Eftekharian S, Seifi S, Satari FD, et al. Curcumin Effect on the Prevention of Gingival Overgrowth Following Phenytoin Consumption in Rats: A Clinicohistological and Immunohistochemical Study. J Contemp Dent Pract 2019;20(10):1146-1150.

Source of support: Babol University of Medical Science

Conflict of interest: None

CCN2 by inhibiting SRC, SMAD3, and JNK receptors. In addition, curcumin reduces the migration of fibroblasts and the expression

(c) The Author(s). 2019 Open Access This article is distributed under the terms of the Creative Commons Attribution 4.0 International License (https://creativecommons. org/licenses/by-nc/4.0/), which permits unrestricted use, distribution, and non-commercial reproduction in any medium, provided you give appropriate credit to the original author(s) and the source, provide a link to the Creative Commons license, and indicate if changes were made. The Creative Commons Public Domain Dedication waiver (http://creativecommons.org/publicdomain/zero/1.0/) applies to the data made available in this article, unless otherwise stated. 
of a-SMA. Therefore, the substance is a potential active ingredient for controlling of gingival volume caused by Phenytoin. ${ }^{4}$

The exact pathogenesis of gingival enlargement caused by Phenytoin and the effect of curcumin in preventing it is still unknown. In this study, weaimed at an accurate evaluation for a better understanding of the pathogenesis and the effect of curcumin in prevention of gingival enlargement using histological, histomorphometric, and immunohistochemical staining. Ki67 (for evaluating cell proliferation) and a-SMA (for evaluating the presence of myofibroblasts) are two of the markers used in immunohistochemical staining. ${ }^{5}$

$\mathrm{Ki} 67$ is a nuclear nonhistone amorphous protein and is a marker of cell proliferation that is expressed in all phases of the cell cycle other than G0 and leads to the coloration of the nucleus in epithelial cells. ${ }^{6}$ a-SMA is a myofibroblast marker, the fibroblasts that have contractile nature of smooth muscles. These are the key cells in wound healing and formation of the fibrous tissue with a key role in inflammation, growth, tissue repair, and cancer. ${ }^{7}$

We used Ki67 and a-SMA markers to identify the curcumin function because Ki67 expresses the characteristics of the epithelium and a-SMA is a marker of the connective tissue.

The objective of the study is to evaluate the clinicohistological and immunohistochemistry effects of curcumin on the gingival enlargement following Phenytoin consumption in rats.

\section{Materials and methods}

The experimental study was approved by our ethical committee then was conducted in Central Animal Housing of Babol Medical University.

In this experimental study, 50 adult male Wistar rats (4 weeks old) were divided into three groups.

\section{Rat Storage Conditions}

The animals were kept in 5 cages, each containing 10 rats, and each rat received a code. The cages were cleaned daily during 57 days of the experiment and the animals received a balanced diet.

The rats in groups I and II received daily Phenytoin $(100 \mathrm{mg} / \mathrm{kg})$ and the animals in group II also received $20 \mathrm{mg} / \mathrm{kg}$ intraperitoneal curcumin per day that was purchased from Merc (Catalog number 820354; chemical formula: $\mathrm{C}_{21} \mathrm{H}_{20} \mathrm{O}_{6}$ ). The control group received the curcumin vehicle (dimethylsulfoxide-DMSO). ${ }^{8}$

\section{Clinical Evaluation}

Measurements were done once at the beginning of the experiments before drug injection and again at the end of the study, 57 days later (1 day after the end of drug injections at the end of the 8th week), based on the Nishikava et al. study. ${ }^{9}$ Photography was performed using a 505 cannon camera available in the periodontology department of the faculty. To calculate the magnification, a gauge (with one hundredth of a millimeter precision) was placed alongside the teeth during photography. The images were imported into the Photoshop software for measurement. The difference between the sizes of MJ-I, the distance between the mucogingival junction and the tooth incisal edge, and GB-I, the distance between the gingival margin edge and the tooth incisal edge of the left mandibular incisal tooth, was indicative of changes in gingival tissue size. Data were analyzed using statistical tests such as ANOVA and the paired $t$ test, where appropriate.

As mentioned, reference points used to determine the difference between the two measurements were MJ-I and the GB-I. ${ }^{9}$

Based on the previous studies, the left mandibular incisal tooth better demonstrates an increase in the volume of gingiva caused by drugs than the rest of the teeth.

\section{Histological, Histomorphometric, and Immunohistochemical Procedures}

All surgical procedures were performed in sterile conditions. After rats were sacrificed, a biopsy was made from the left incisal gingiva (from mid-papillary to distal and from the coronal section to the mucogingival fold). The tissue samples were fixed immediately in $10 \%$ formalin. Samples were sent to a pathology lab and immersed in formalin for 48 hours. Finally, the specimens were divided into two sections in the direction of the largest cross-section, and their paraffin blocks were prepared. Serial sections $(5 \mu \mathrm{m})$ were prepared from each paraffin block. One section was stained with hematoxylin-eosin staining and two other sections with immunohistochemical staining.

The histopathologic study of the hematoxylin-eosin sections was performed using optical microscopy (Olympus Bx41, Japan) at $40 \times$ magnification in five serial fields and the inflammation rate in the specimens was determined at 4 degrees as follows: ${ }^{10}$

Grade 0: The absence of inflammatory cells

Grade I: Each microscopic field contained 0-10 inflammatory cells (mild)

Grade II: Each field contained 10-50 inflammatory cells (moderate)

Grade III: More than 50 inflammatory cells were present in each field (severe)

\section{Immunohistochemical Staining}

Five micron sections were prepared from the selected blocks and immunohistochemistry staining was performed using the standard avidin biotine peroxidase method. ${ }^{5,6}$

Positive, immunohistochemical staining with a-SMA and Ki67 of breast ductal carcinoma and negative, nonimmunized rat serum without primary antibody controls were performed along with the sections to evaluate the accuracy and validity. The endothelial staining of the blood vessel walls with a-SMA in normal oral mucosa was considered as the positive internal control. All the stained slides were examined by an oral pathologist using an Olympus optical microscope with a magnification of $40 \times$.

The cytoplasm of myofibroblasts colored with a-SMA and the nucleus of the epithelial cells colored with Ki67 were considered as positive.

\section{Immunohistochemical Evaluation}

For immunohistochemical evaluatuion staining with a-SMA, the samples were counted in 10 microscopic fields (40X), and number of nucleus epithelial stained cells in 1,000 cells was considered as percentage. $^{6}$

The a-SMA samples were scored as follows:

Score (1): Myofibroblasts were not stained with a-SMA or if less than $1 \%$ of myofibroblasts were stained with a-SMA.

Score (2): If more than $1 \%$ and less than $50 \%$ of myofibroblasts were stained with a-SMA.

Score (3): If more than $50 \%$ myofibroblasts were stained with a-SMA.

For Ki67 staining, 10 microscopic fields (40x) were selected and the number of nucleus epithelial stained in 1,000 cells was considered as percentage. ${ }^{5}$

\section{Histomorphometric Evaluation}

All prepared sections from each biopsy were photographed by a digital camera mounted over an Olympus microscope (40x), 
and the resulting images (in the jpeg format) were imported into the Motic plus2 (Micro-optic industrial group Co., Ltd.) software. Separate epithelium and connective images were prepared for the evaluations.

\section{Determining the Thickness of the epithelium}

Following serial sectioning in the paraffin blocks, the first, second, and sixth sections of each group were selected and images were prepared at the magnification of $10 \times$. The images were imported into the Motic plus2 software and the average thickness of the epithelium in three different regions from surface to the basal lamina was calculated as the thickness of the epithelium. ${ }^{11}$

\section{Determining the Number and Cross Section of Blood Vessels}

An oral pathologist, blind to the status of the numbered slides, examined the samples at $10 \times$ and photographed $(40 \times)$ three regions with the maximum number of blood vessels. The images were imported into the Motic Plus2 software and the number and cross-sectional area of the blood vessels in each field were recorded and the mean value was calculated. ${ }^{12}$

\section{Results}

The ANOVA test revealed no significant difference in the mean of gingival dimensions in the three groups before the intervention $(p=0.9)($ Fig. 1A).

The paired $t$ test was used to compare the gingival dimensions before and after the intervention in the three groups and the results are as follows:
In group I, injected with Phenytoin, gingival volume increased by $0.5 \mathrm{~mm}(p=0.002)$. However, in the control group, which did not receive Phenytoin, increase in the gingival volume was very small $(0.13 \mathrm{~mm})$ and was not statistically significant $(p=0.135)$.

In group III, which received curcumin in addition to Phenytoin, similar results to the control group $(0.15 \mathrm{~mm})$ were observed, indicating that no increase in gingival volume had happened during the treatment ( $p=0.195$ ) (Table 1).

The results of the repeated measure ANOVA showed that the intervention was effective $(p=0.002)$.

During the 8 weeks of this study, four rats in group II (Phenytoin-curcumin) and one rat in the control group died.

Hematoxylin-eosin and morphometry results were summarized in Table 2 (Figs $1 \mathrm{~B}$ and $\mathrm{C}$ ).

The difference in the expression of Ki67 and a-SMA between group I and group II was statistically significant (Table 3) (Figs 1D and $\mathrm{E})$.

\section{Discussion}

This was the first study in the animal model that evaluated the effect of curcumin in preventing gingival volume increase after Phenytoin consumption in rats. In group I, injected with phenytoin, gingival volume increased; however, in the control group, which did not receive Phenytoin, increase in the gingival volume was very small $(0.13 \mathrm{~mm})$ and was not statistically significant. Thus, Phenytoin increased gingival volume in rats and the increase was not due to other factors such as normal growth with age.

In group II, which received curcumin in addition to phenytoin, similar results to the control group $(0.15 \mathrm{~mm})$ were observed,

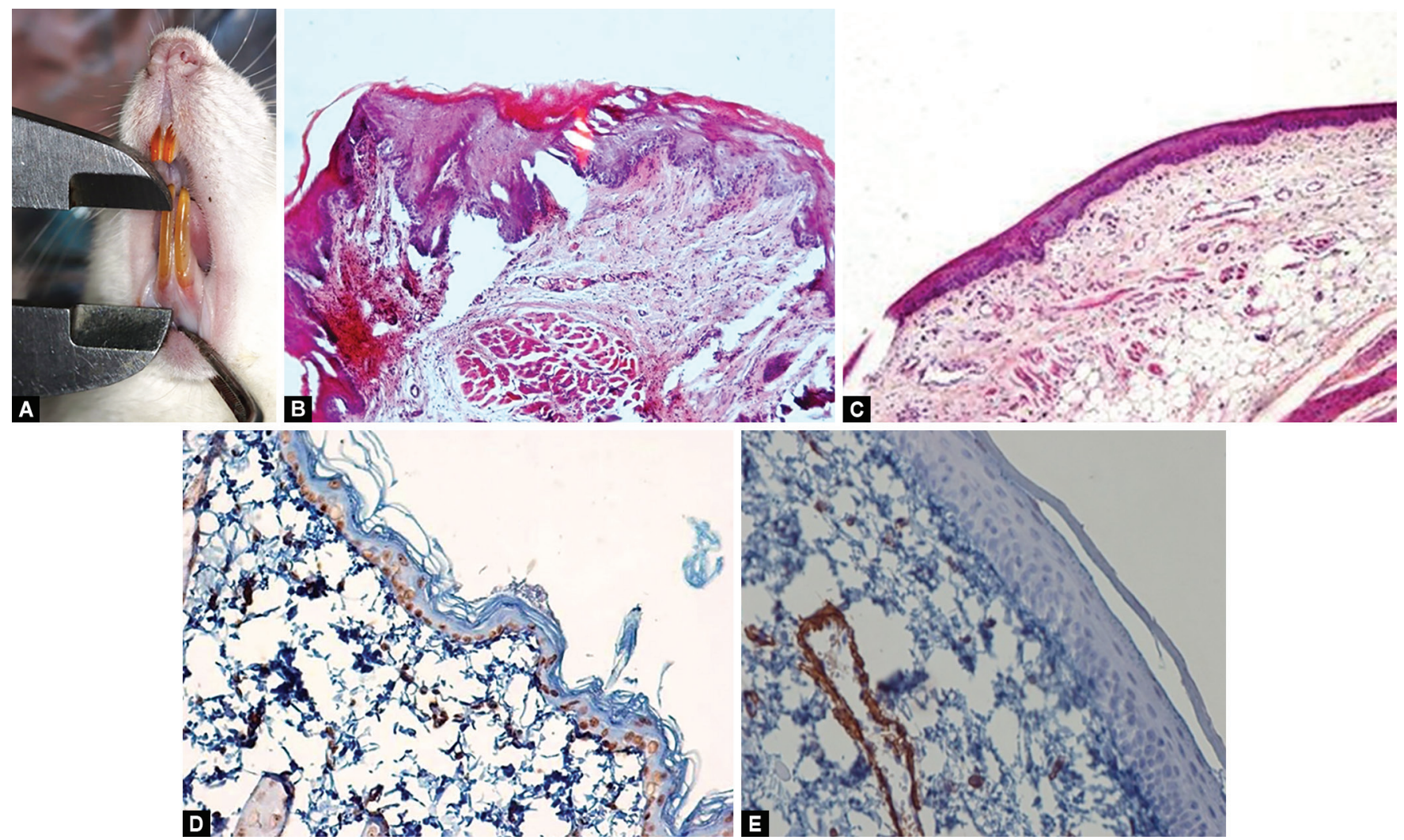

Figs $1 \mathrm{~A}$ to $\mathrm{E}$ : (A) Clinical view, reference point in rat; (B) Hematoxylin-eosin staining $(\times 10)$, increase of epithelial thickness in group I; (C) Hematoxylineosin staining $(\times 10)$, reduction of epithelial thickness in group II; (D) Immunohistochemical staining with Ki67 in group I, Ki67, a-SMA positive staining (X10); (E) Immunohistochemical staining with Ki67 and a-SMA in group II ( $\times 10)$ — no staining of Ki67, only stained vessel with a-SMA 
Curcumin Effect on the Prevention of Gingival Overgrowth

Table 1: Gingival measurements in different groups before and after the intervention

\begin{tabular}{lllll}
\hline $\begin{array}{l}\text { Average measured gingival } \\
\text { dimensions }\end{array}$ & $\begin{array}{l}\text { Before intervention } \\
(\mathrm{mm})\end{array}$ & $\begin{array}{l}\text { After intervention } \\
(\mathrm{mm})\end{array}$ & $\begin{array}{l}\text { Increase in gingival } \\
\text { volume }(\mathrm{mm})\end{array}$ & $\begin{array}{l}p \text { value }(\mathrm{pairedt} \\
\text { test })\end{array}$ \\
\hline Control group & 1.68 & 1.81 & 0.13 & 0.135 \\
Group I (Phenytoin) & 1.48 & 1.98 & 0.5 & 0.002 \\
Group II (Phenytoin + curcumin) & 1.63 & 1.78 & 0.15 & 0.195 \\
$p$ value & 0.204 & & & \\
\hline
\end{tabular}

Table 2: Results of the hematoxylin-eosin staining and morphometry

\begin{tabular}{|c|c|c|c|c|}
\hline Group & Inflammation grade & $\begin{array}{l}\text { Mean number of } \\
\text { vessels }\end{array}$ & $\begin{array}{l}\text { Mean cross-sectional } \\
\text { area of the vessels }(\mu \mathrm{m})\end{array}$ & $\begin{array}{l}\text { Epithelium mean } \\
\text { thickness }(\mu \mathrm{m})\end{array}$ \\
\hline \multirow[t]{2}{*}{ Control } & No inflammation (6 samples) & $2.44 \pm 1.014$ & $410.03 \pm 1.4$ & $540.04 \pm 0.6$ \\
\hline & Mile (3 samples) & & & \\
\hline \multirow[t]{3}{*}{ Group I (Phenytoin) } & Mild (3 samples) & $5.89 \pm 1.844$ & $1930.08 \pm 1.9$ & $2298.009 \pm 1.05$ \\
\hline & Moderate (7 samples) & & & \\
\hline & Severe (10 samples) & & & \\
\hline $\begin{array}{l}\text { Group II (Phenytoin + } \\
\text { curcumin) }\end{array}$ & Mild (15 samples) moderate (1 sample) & $2.79 \pm 1.424$ & $535.202 \pm 1.2$ & $734.407 \pm 1$ \\
\hline$p$ value & 0.003 & 0.001 & 0.001 & 0.002 \\
\hline
\end{tabular}

Table 3: Immunohistochemical staining findings in the three groups

\begin{tabular}{lcl}
\hline Group & Mean score, Ki67 & Mean score, a-SMA \\
\hline Control & $6.6 \%$ & Score 1 \\
Group I (Phenytoin) & $60.4 \%$ & Score 3* \\
Group II (Phenytoin + curcumin) & $8.9 \%$ & Score 2 \\
\hline
\end{tabular}

$$
{ }^{*} p=0.001
$$

indicating that no increase in gingival volume had happened during the treatment $(p=0.195)$

In the control group, which received the vehicle of curcumin, gingival enlargement was not seen; therefore, the vehicle of curcumin is not effective in gingival enlargement.

Today, the use of herbal remedies has been more in the focus due to their lower cost and fewer side effects in comparison with chemical drugs. Curcumin is the active ingredient derived from turmeric with antioxidant, antitumoral, and anti-inflammatory properties. ${ }^{13}$ The precise cellular and molecular mechanisms of the gingival enlargement caused by Phenytoin have not been fully understood till now. So, we aimed at an accurate evaluation for a better understanding of the functional mechanisms of curcumin using histopathological evaluations including hematoxylin-eosin and immunohistochemical staining with Ki67 and a-SMA and morphometry. In the histological and morphometric evaluation, in group II (curcumin + Phenytoin), decrease in the number of blood vessels, reduction of inflammation, and reduction of epithelial thickness were observed in the pathology view compared to group I (Phenytoin). Murgana et al. also investigated the effect of curcumin in rats with periodontal disease and reported that curcumin significantly reduced inflammatory infiltration and increased the collagen content. ${ }^{14}$ Savita et al. reported that nanocurcumin reduced proinflammatory cytokines and elevated levels of antioxidants in the liver and inhibited fibrosis induced by the activated myofibroblasts, ${ }^{15}$ which are in agreement with our study. In the present study, it seems that curcumin exerts its effects by reducing inflammatory cytokines like TNF- $a, I L-1$, and IL-8 and increasing the expression of fibrotic cytokines such as TGF- $\beta^{5,6}$ which led to a reduction in inflammatory infiltration and a decrease in the number of blood vessels. We observed an inverse relationship between the number of blood vessels and their cross-sectional area so that the highest number of blood vessels and the lowest cross-sectional area were observed in group I (Phenytoin). It seems that curcumin exerts its effects by reducing the number of blood vessels and increasing their cross-sectional area as compared to the control group. Ki67 is a nuclear nonhistone amorphous protein, which is expressed in all phases of the cell cycle other than $\mathrm{G} 0$ and can stain the nucleus of the cell. ${ }^{6}$ a-SMA indicates the number of myofibroblasts or fibroblasts with smooth muscle contraction, which plays a major role in wound healing. ${ }^{5}$ We observed that the expression of Ki67 and a-SMA in group II was significantly lower than group I, which indicates curcumin mechanism of action. According to the epithelial-mesenchymal transition, all changes in epithelium were terminated connective tissue change. ${ }^{16}$ In the study, increase proliferation of epithelium was caused by connective tissue activity specially myofibroblast action. The histological findings of the present study confirm its clinical findings and in some way the histological results represent the accuracy of the clinical results of the study in identifying curcumin mechanism and function. Hematoxylin-eosin is the best staining for diagnosis in histology, but immunohistochemistry is a helpful method in understanding of curcumin functional mechanism. ${ }^{17}$

Chen et al. have shown that curcumin inhibits thrombininduced CCN2 effects and has an inhibitory effect on human gingival fibroblasts. ${ }^{3}$ In the current study, the application of Phenytoin during 8 weeks resulted in a significant increase in gingival volume in rats. Also, curcumin was effective in preventing the increase in gingival volume caused by phenytoin. The results of this study are in line with the results of previous studies, in which 
curcumin reduced the TGF- $\beta$ level by inhibiting SRC, SMAD3, and JNK receptors. In addition, curcumin reduced myofibroblasts activation and the expression of a-SMA. Therefore, curcumin is effective in preventing gingival overgrowth caused by Phenytoin.

Chen et al. reported that curcumin prevents the expression of thrombin-induced CCN2 by inhibiting JNK in human gingival fibroblasts. Curcumin reduces the formation and development of fibrosis and reduces the release of TIMP-1 and oxidative stress in satellite cells in mice. It can also reduce the accumulation of myofibroblastic hepatic stellate cells (MHSCs) and myofibroblastic hepatocyte cells by decreasing the expression of a-SMA in mice, which indicates an antifibrotic effect of curcumin. ${ }^{3}$

In this experimental study, adult male Wistar rats (4 weeks old) were used. This model for evaluating the gingival enlargement, which is based on the Tamamori study, ${ }^{18}$ has several advantages. Other animal species used in previous studies included monkeys and cats that in addition to higher costs required a longer period of Phenytoin injection (3-5 month) for creating an increase in gingival volume. The use of rats is more suitable because of the availability of the animal and the lower cost and low differences in the response to the administration of the drug in each strain that produces better results over repeating the experiments. According to previous studies by Tamamori, younger rats (15 days) showed a greater increase in gingival volume than the older ones (45 days) over a 40-day phenytoin application. Therefore, it was suggested to start the drug application at an earlier age, but some studies have found this to be unfeasible as the administration of Phenytoin at an early age (infancy) represses the central nervous system of the rat and leads to animal death. Based on Nashikava et al. ${ }^{9}$ study, Phenytoin dose at the end of 8 weeks was $80-100 \mathrm{mg} / \mathrm{kg}$. Measuring the animal's weight at the beginning and end of the study, we witnessed a normal growth of the animal. ${ }^{18}$

Administration of $20 \mathrm{mg} / \mathrm{kg}$ of curcumin over a period of 8 weeks in this study caused no significant mortality in rats and seems to be a suitable dose for administration.

According to the Yang's study, curcumin may prevent recurrence increase in gingival volume after surgery as it suppresses CCN2 induced by TGF- $\beta 1$ through inhibiting JNK, SRC, and Smad3 receptors in gingival fibroblasts. ${ }^{4}$ We suggest further investigation of the curcumin effect in preventing recurrence increase in volume after gingival surgery. This is a preliminary study that aims at the assessment of curcumin in preventing of gingival enlargement in rats for the first time. Based on this discussion, it can be concluded that curcumin is effective in preventing of gingival overgrowth after Phenytoin consumption; however, larger sample size and larger clinical trials are required to arrive at a definitive conclusion.

\section{Conclusion}

Curcumin seems to uses its effects in preventing of gingival enlargement caused by Phenytoin through decreasing the inflammatory infiltration, decreasing the number of blood vessels and increasing their cross-sectional area, decreasing the thickness of the epithelium, and decreasing the expression of Ki67 and a-SMA in rats.

\section{Acknowledgments}

We thank Mr Sheikhzadeh and Mr Noori for helping in laboratory.

\section{References}

1. Newman MG, Takei HH, et al. Carranza's clinical periodontology. 12th ed., Philadelphia: Saunders; 2015. pp 103-105.

2. Black S, Palamakumbura A, et al. Tissue-specific Mechanisms for CCN2/CTGF Persistence in Fibrotic Gingiva: interactions between CAMP and MAPK signaling pathways, and prostaglandin E2-EP3 receptor mediated activation of the c-JUN N-terminal kinase. J Biol Chem 2007 May;282(21):15416-15429. DOI: 10.1074/jbc. M610432200.

3. Chen $Y$, Yang $W$, et al. Curcumin Inhibits Thrombin-Stimulated Connective Tissue Growth Factor (CTGF/CCN2) Production Through c-Jun NH2-Terminal Kinase Suppression in Human Gingival Fibroblasts. J Periodontol 2012 Dec;83(12):1546-1553. DOI: 10.1902/ jop.2012.110641.

4. Yang W-H, Kuo M-P, et al. Curcumin Inhibits TGF $\beta 1$-induced CCN2 via Src, JNK, and Smad3 in Gingiva. J Dent Res 2013 Jul;92(7):629-634. DOI: 10.1177/0022034513488139.

5. Seifi S, Shafaei SH, et al. Myofibroblast stromal presence and distribution in squamous epithelial carcinoma,oral dysplasia and hyperkeratosis. Asian Pac J Cancer Prev 2010 Dec;11(2): 359-364.

6. Salva Guegel GA, Goncalres Ramos EA, et al. Expression of ki 67 , p53 and p53 protein in keratocyctic odontogenic tumors: an immunohistochemical study. J Mol Histol 2008 Jun;39(3):311-316. DOI: 10.1007/s10735-008-9167-0.

7. Tusgino T, Seshimo I, et al. Stromal myofibroblasts predict disease recurrence for colorectal cancer. Clin Cancer Res 2007 Apr;13(7): 2082-2090. DOI: 10.1158/1078-0432.CCR-06-2191.

8. Okuneif $\mathrm{P}, \mathrm{Xu}$ J, et al. Curcumin Protects Against Radiation-Induced Acute And Chronic Cutaneous Toxicity in Mice And Decreases mRNA Expression of Inflammatory and Fibrogenic Cytokins. Int J Radiat Oncol Biol Phys 2006 Jul;65(3):890-898. DOI: 10.1016/ j.ijrobp.2006.03.025.

9. Nashikava S, Nagata T, et al. Pathogenesis of drug-induced gingival overgrowth. A review of studies in the rat model. J Periodontol 1996 May;67(5):463-471. DOI: 10.1902/jop.1996.67.5.463.

10. Rokn AR, Khodadostan MA, et al. Bone formation with two types of grafting materials: a histologic and histomorphometric study. Open Dent J 2011 Jul;5:96-104. DOI: 10.2174/1874210601105010096.

11. Bulut $\mathrm{S}, \mathrm{Uslu} \mathrm{H}$, et al. Analysis of proliferation activity in oral gingival epithelium in immunosuppressive medication induced gingival overgrowth. Head Face Med 2006 May;2:13. DOI: 10.1186/1746-160X2-13.

12. Seifi $S$, Shafaie $S$, et al. Microvessel density in follicular cysts, keratocystic odontogenic tumours and ameloblastomas. Asian Pac J Cancer Prev 2011;12(2):351-356.

13. Troijilo J, Irasema Y. Renoprotective of the effect of curcumine. Epilepsy Res 2011 Jul;26(1):207-218.

14. Morgana R, Sabrina G, etal.Curcumin modulates the immune response associated with LPS-induced periodontal disease in rats. Innate Immunity 2012 Feb;18(1):155-163. DOI: 10.1177/1753425910392935.

15. Bisht $S$, Muzuma $M$, et al. Systemic administration of polymeric nanoparticle-encapsulated curcumin (NanoCurc ${ }^{\mathrm{TM}}$ ) blocks tumor growth and metastases in preclinical models of pancreatic cancer. Mol Cancer Ther 2010 Aug;9(8):2255-2264. DOI: 10.1158/1535-7163. MCT-10-0172.

16. Sume SS, Kantarci A, et al. Epithelial to mesenchymal transition in gingival overgrowth. Am J Pathol 2010;177:208-218. DOI: 10.2353/ ajpath.2010.090952.

17. John KC, Chan MD. The wonderful colors of the hematoxylin-eosin stain in diagnostic surgical pathology. Int J Surg Pathol 2014;22(1): 12-32. DOI: 10.1177/1066896913517939.

18. Tamamori Y, Tamura Y, et al. Establishment of Rat Model of DrugInduced Gingival Overgrowth Induced by Continuous Administration of Phenytoin. J Pharmacol Sci 2005 Jul;98:290-297. DOI: 10.1254/jphs. FPJ04028X. 